\title{
A Utility-Based Routing Scheme in Ad Hoc Networks
}

\author{
Jie $\mathrm{Wu}$ \\ National Science Foundation, USA \\ jwu@nsf.gov
}

\begin{abstract}
To efficiently address the routing problem in ad hoc networks, we introduce a new utility metric, maximum expected social welfare, and integrate the cost and stability of nodes in a unified model to evaluate the optimality of routes. The expected social welfare is defined in terms of expected benefit (of the routing source) minus the expected costs incurred by forwarding nodes. Based on this new metric, we design an optimal and efficient algorithm, and implement the algorithm in both centralized (optimal) and distributed (near-optimal) manners. We also look at several extensions in improving benefit, all optimal routes, incentive compatible routing, and applications of this new model.
\end{abstract}

